\title{
Intermittent pneumatic compression is a cost- effective method of orthopedic postsurgical venous thromboembolism prophylaxis
}

This article was published in the following Dove Press journal:

ClinicoEconomics and Outcomes Research

\author{
Rhodri Saunders' \\ Anthony J Comerota ${ }^{2}$ \\ Audrey Ozols ${ }^{3}$ \\ Rafael Torrejon Torres' \\ Kwok Ming $\mathrm{Ho}^{4}$ \\ 'Coreva Scientific, Freiburg, Germany: \\ ${ }^{2}$ Jobst Vascular Institute, Toledo, $\mathrm{OH}$, \\ USA; ${ }^{3}$ Medtronic, Boulder, CO, USA; \\ ${ }^{4}$ Royal Perth Hospital and School \\ of Population Health, University \\ of Western Australia, Perth, WA, \\ Australia
}

Correspondence: Rhodri Saunders Coreva Scientific, Kaiser-Joseph-Strasse 198-200, 79098 Freiburg, Germany Tel +4976176999422

Fax +49 76I 76999421

Email rhodri@coreva-scientific.com
Background: Venous thromboembolism (VTE) is a major complication after lower-limb arthroplasty that increases costs and reduces patient's quality of life. Using anticoagulants for 10-35 days following arthroplasty is the standard prophylaxis, but its cost-effectiveness after accounting for bleeding complications remains unproven.

Methods: A comprehensive, clinical model of VTE was created using the incidences, clinical effects (including bleeding), and costs of VTE and prophylaxis from randomized controlled trials, meta-analyses, and large observational studies. Over 50 years, the total health care costs and clinical impact of three prophylaxis strategies, that are as follows, were compared: low-molecular-weight heparin (LMWH) alone, intermittent pneumatic compression (IPC), and IPC with LMWH (IPC+LMWH). The cost per VTE event that was avoided and cost per quality-adjusted life year (QALY) gained in both the US and Australian health care settings were calculated.

Results: For every 2,000 patients, the expected number of VTE and major bleeding events with LMWH were 151 and 6 in the USA and 160 and 46 in Australia, resulting in a mean of 11.3 and 9.1 QALYs per patient, respectively. IPC reduced the expected VTE events by 11 and 8 in the USA and Australia, respectively, compared to using LMWH alone. IPC reduced major bleeding events compared to LMWH, preventing 1 event in the US and 7 in Australia. IPC+LMWH only reduced VTE events. Neither intervention substantially impacted QALYs but both increased QALYs versus LMWH. IPC was cost-effective followed by IPC+LMWH. Conclusion: IPC and IPC+LMWH are cost-effective versus LMWH after lower-limb arthroplasty in the USA and Australia. The choice between IPC and IPC+LMWH depends on expected bleeding risks.

Keywords: VTE, IPC, thromboprophylaxis, arthroplasty, mechanical prophylaxis, cost-effectiveness

\section{Background}

Venous thromboembolism (VTE), including deep vein thrombosis (DVT) and pulmonary embolism (PE), is a serious condition that affects both patient outcomes directly and the overall health care utilization. Globally, there are about 10 million acute VTE events every year and it was estimated that 0.95 million adults in the USA live with VTE. ${ }^{1,2}$ VTE is associated with a wide range of undesirable patient outcomes including respiratory and cardiovascular failure and prolonged hospital stay, and it is considered one of the leading causes of preventable in-hospital death. ${ }^{1}$ VTE also has long-term consequences, in particular postthrombotic syndrome (PTS), resulting in continued patient discomfort and increased long-term health care utilization. 
A conservative estimate has placed the annual economic burden of VTE in the USA at 7-10 billion USD in 2014; the mean direct costs of care per patient who survived the VTE event were estimated to be between 18,000 and 23,000 USD. ${ }^{3}$ VTE is a global disease. Barco et al analyzed data from 28 countries of European Union, finding that the total annual direct burden of VTE was in the range of $€ 3.0-8.5$ billion in 2014, of which the authors estimated $€ 2.2-6.2$ billion was avoidable. ${ }^{4}$ The rate of VTE in Australia is recognized to be higher than in other health care settings. ${ }^{5}$ The cost per VTE event in Australia was estimated to be 10,007 AUD, and when considering lost productivity, VTE was one of the most costly, but preventable, conditions in Australia. ${ }^{6}$

Surgery, particularly in lower limb arthroplasty, puts patients at very high risk of developing VTE. In patients, after lower limb arthroplasty, all three major pathogenic elements of VTE - venous stasis, vessel wall injury, and hypercoagulability - are likely activated. Rates of VTE after total knee arthroplasty (TKA) and total hip arthroplasty (THA) have been reported to be between 40 and 94 and 13 and 24 per 1,000 patients, respectively. ${ }^{7-9}$ With an aging population, the overall incidence and impact of VTE after arthroplasty are expected to increase substantially over the next few decades. For instance, the number of THA and TKA procedures have been increasing at 3.7\% and 7.0\% per year in Australia. ${ }^{10}$ Each year, postsurgical VTE adds approximately 66 million AUD to the cost of care for THA and TKA. ${ }^{11}$ Beyond financial costs, VTE after arthroplasty also has an impact on patients' quality of life (QoL) due to symptoms of PTS after DVT and respiratory insufficiency after severe PE. Estimates from the Euro QoL 5-dimensions (EQ-5D) health survey indicates that patients lose between $1 \%$ and $48 \%$ of their QoL after a VTE event. ${ }^{12-14}$

Currently, using anticoagulants for 10-35 days to reduce hypercoagulability and VTE after lower limb arthroplasty is considered the standard of care. ${ }^{15,16}$ Due to its long history of use and a lower cost compared to direct oral anticoagulants, low-molecular-weight heparin (LMWH) remains the most common anticoagulant used for VTE prophylaxis in many surgical patients including those who underwent arthroplasty. ${ }^{15,16}$ Because all anticoagulants have an inherent risk of inducing bleeding, an assessment of bleeding risk in patients should occur before determining whether anticoagulants should be started. ${ }^{15}$ Trials indicate that up to $9.9 \%$ of patients using anticoagulant prophylaxis experience some form of bleeding events. ${ }^{7,17}$ Bleeding events after use of anticoagulants can be costly and have significant impact on patient's QoL. Although uncommon, even at therapeutic doses of heparin, intracranial hemorrhage (ICH) may have catastrophic consequences for patients, with substantial reduction in QoL. ${ }^{18,19}$ The mean direct cost of an ICH was found to be 35,931 USD, and subsequent care costs in the years after the event averaged 16,254 USD. ${ }^{20}$

An alternative approach to VTE prophylaxis is to employ mechanical means either alone or in combination with anticoagulants simultaneously or sequentially. Intermittent pneumatic compression (IPC) of the lower limbs improves venous blood flow velocity in a cyclical fashion similar to ambulation. There is also evidence to suggest that IPC may promote fibrinolysis activity and hence reduces the risk of lower limb VTE without increased risk of bleeding. ${ }^{9,21}$ Data from a large number of randomized controlled trials (RCTs) showed that IPC is effective and possibly comparable to anticoagulants in reducing VTE in hospitalized patients. ${ }^{23,27}$ In the specific subgroup of patients after arthroplasty, IPC also appears to be as effective as anticoagulants, albeit the number of RCTs comparing different strategies is more limited..$^{24,27}$

We hypothesized that because IPC is associated with a reduced risk of bleeding and possibly equally effective in reducing VTE compared to anticoagulants, IPC may be more cost-effective than LMWH in preventing VTE after lowerlimb arthroplasty. In this study, we developed a comprehensive model to analyze the clinical and economic outcomes and assess the cost-effectiveness of three possible VTE prophylaxis strategies (LMWH alone, IPC alone, or a combination of IPC and LMWH [IPC+LMWH]) after arthroplasty applied to both the US and Australian health care settings.

\section{Methods}

From the perspective of health care payers, the cost and effects of interventions are not limited to a patient's time in hospital or time on treatment, but rather are measured over decades. As it is rarely feasible to perform such long-term studies in realworld practice, comprehensive computational models of VTE and VTE prophylaxis were developed. Therefore, two Markov models were developed by the authors to estimate events, outcomes, and costs following lower-limb arthroplasty from 1 to 50 years. For VTE, the events and outcomes (health states) included were: "no VTE," "DVT," "PE," "DVT+PE," "previous VTE," "recurrent VTE," "postthrombotic syndrome," "VTE death," and "death." These health states and the transitions allowed between them are depicted in Figure 1A. All patients started in the "No VTE" state, and the probabilities for moving to another state were taken from peer-reviewed, published literature (Table 1) reporting on patients receiving LMWH as prophylaxis. The meta-analysis published by Ho 
et al provided the relative risk (RR) of events and outcomes for IPC and IPC+LMWH (Table 1). ${ }^{22}$

The second part of the model estimated adverse events associated with VTE prophylaxis (Figure 1B). The events and outcomes included were "no event," "minor bleed," "wound infection," "heparin-induced thrombocytopenia," "ICH," "other major bleed," "previous major bleed," and "major bleed death." The probabilities of transitioning between VTE prophylaxis health states were also taken from peer-reviewed literature (Table 1) and were specific to use of LMWH. Events and outcomes for IPC and IPC+LMWH were estimated by using RRs taken from the meta-analyses of Ho et $\mathrm{al}^{22}$ and O'Connell ${ }^{29}$ (Table 1). Running the model with LMWH data provided VTE events and outcomes for patients receiving LMWH prophylaxis. A subsequent run of the model with LMWH data and the RR data for IPC provided VTE events and outcomes for patients receiving IPC prophylaxis. Costeffectiveness was determined by comparison of outcomes between the two model runs.

A model run consists of multiple iterations or cycles of the Markov models described. Each cycle represents a discrete period of time, and during the first month, the model cycle length was 1 day. After this, monthly cycles were performed up to 6 months. From this point on, half yearly cycles were used. Over 50 years, patient events and outcomes were therefore assessed 130 times. In data presented in this study, the VTE model ran before the VTE prophylaxis model. The proportion of patients alive at the start of the VTE prophylaxis model must equal that at the end of the VTE model and vice versa. Patients who die in one model are, thus, removed from the second model, for example, 1/7 of the proportion of patients who died ("death" or "VTE death")

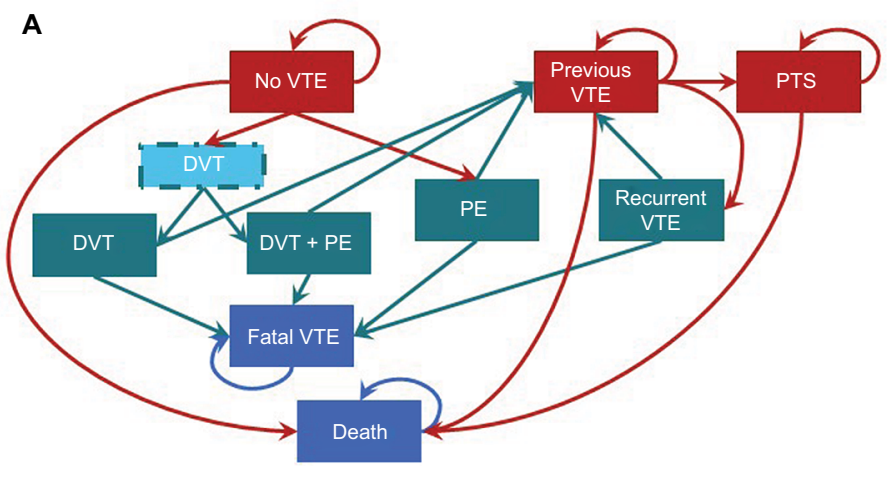

B

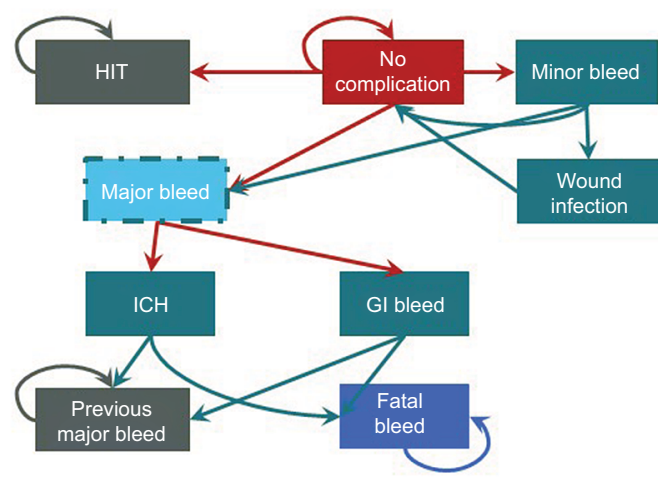

Recurring health state

Event i.j Transient event decision

Fatal state

Figure I Depiction of the two Markov models for (A) VTE and (B) VTE prophylaxis.

Abbreviations: DVT, deep-vein thrombosis; GI, gastrointestinal; HIT, heparin-induced thrombocytopenia; ICH, intracranial hemorrhage; PE, pulmonary embolism; PTS, postthrombotic syndrome; VTE, venous thromboembolism.

Table I Incidence of adverse events during VTE prophylaxis

\begin{tabular}{|c|c|c|c|c|}
\hline Events & $\begin{array}{l}\text { Incidence USA } \\
\text { (per days) }\end{array}$ & $\begin{array}{l}\text { Incidence Australia } \\
\text { (per days) }\end{array}$ & $\begin{array}{l}\text { RR with IPC } \\
(95 \% \mathrm{CI})\end{array}$ & $\begin{array}{l}\text { RR with IPC+LMWH } \\
(95 \% \mathrm{CI})\end{array}$ \\
\hline DVT & $0.55 \%(3)^{23}$ & $4.48 \%(\mathrm{II} .52)^{24}$ & $0.86(0.56-|.3|)^{22}$ & $0.32(0.16-0.64)^{22}$ \\
\hline PE & $0.39 \%(3)^{23}$ & $0.25 \%(11.52)^{24}$ & $0.99(0.36-2.76)^{22}$ & $0.62(0.13-3.02)^{22}$ \\
\hline VTE recurrence & $14.3 \%(365.25)^{25}$ & $1.9 \%(90)^{26}$ & & \\
\hline Recurrent VTE that is PE & $20.5 \%{ }^{25}$ & $20 \%{ }^{26}$ & & \\
\hline Non-major bleed & $9.9 \%(42)^{7}$ & $9.9 \%(12)^{17}$ & $0.41(0.26-0.65)^{22}$ & $\mathrm{I}^{\mathrm{a}}$ \\
\hline Major bleed & $0.26 \%(9)^{27}$ & $1.9 \%(90)^{28}$ & $0.85(0.74-0.97)^{29}$ & $I^{a}$ \\
\hline Major bleed that is $\mathrm{ICH}$ & $7.5 \%{ }^{12}$ & $4 \%^{30}$ & & \\
\hline Wound infection & $2.6 \%(30)^{7}$ & $5.2 \%(30)^{11}$ & $\mathrm{I}^{\mathrm{a}}$ & $\mathrm{I}^{\mathrm{a}}$ \\
\hline Wound infections that are deep & $34.62 \%^{7}$ & $30.77 \%{ }^{11}$ & & \\
\hline Heparin-induced thrombocytopenia & $0.04 \%(4)^{31}$ & $0.2 \%(6.55)^{28}$ & $0^{\mathrm{b}}$ & $\mathrm{I}^{\mathrm{a}}$ \\
\hline PTS & $22.8 \%(730.5)^{32}$ & $22.8 \%(730.5)^{32}$ & & \\
\hline Severe PTS & $10 \%{ }^{33}$ & $10 \% 33$ & & \\
\hline Mortality & From US lifetables & From Australian lifetables & $0.92^{22}$ & $0.6 I^{22}$ \\
\hline
\end{tabular}

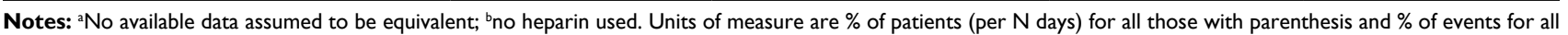
those without parenthesis.

Abbreviations: DVT, deep vein thrombosis; ICH, intracranial hemorrhage; IPC, intermittent pneumatic compression; LMWH, low-molecular-weight heparin; PE, pulmonary embolism; PTS, postthrombotic syndrome; RR, risk ratio; VTE, venous thromboembolism. 
were removed from each of the seven alive states in the VTE prophylaxis model. Likewise, $1 / 7$ of the proportion of patients who died (major bleed death) were removed from each of the seven alive states in the VTE model. The model order was exchanged to test the impact of structural uncertainty, and the model outcomes were not substantially different. The model development followed good practice guidance from the International Society for Pharmacoeconomics and Outcomes Research. ${ }^{34-36}$

\section{Model input data}

Peer-reviewed literature for informing the model design and providing input data were identified through structured review of PubMed. The search identified indexed abstracts containing target words and/or phrases in their title, abstract, key words, or Medical Subject Headings (MeSH) terms. The aim of this study was to find high-quality data on the safety and efficacy of VTE prophylaxis and costs and QoL data related to VTE, VTE prophylaxis, or their associated events and outcomes. The search was first performed in 2015 and repeated periodically thereafter to capture newly indexed abstracts.

Literature review determined that common risk factors for VTE are age ( $>80$ years), female gender, BMI (>30 kg/ $\mathrm{m}^{2}$ ), and previous VTE. ${ }^{37}$ In the countries of interest, the percentage of lower-limb arthroplasties that are of the hip rather than the knee is $33.0 \%$ and $43.5 \%$ in the USA and Australia, respectively. ${ }^{10,38}$ Guideline prophylaxis use is between 10 and 30 days following lower-limb arthroplasty. ${ }^{15,16}$ Prophylaxis of interest was LMWH, IPC, and IPC+LMWH. IPC+LMWH was considered to be combined use of the two products during the time in hospital, followed by LMWH alone after discharge. The cost of prophylaxis included acquisition, setup, and administration. Prophylaxis impacted QoL via injection requirement, restricted movement, and fear of bleeding.

Costs were presented with their corresponding values in 2015 (USD and AUD). Costs from another year were converted to 2015 values by using health care-specific inflation indices. Peer-reviewed literature provides the majority of costs for the US setting. For Australia, costs from the Australian refined diagnosis-related group public hospital refund scheme were used and supplemented with published data (Table 2). All costs are from the payer perspective and do not include societal costs such as time off work. The annual discount rate used after 1 year was $3.5 \%$ in the USA and $5.0 \%$ in Australia.

Quality-adjusted life expectancy (QALE), measure in quality-adjusted life years (QALYs), for the patient population and for each adverse event was taken from published literature where available (Table 2), with preference given to articles reporting on EQ-5D scores. Where utilities were unavailable, estimates were made by calculating the annual amount of perfect QoL expected to be lost. As a year of perfect QoL is worth 1, every hour of QoL lost is a deficit of 0.000114 QALYs.

\section{Base case settings}

The cost-effectiveness of VTE prophylaxis was assessed over a 50-year time period in terms of the cost per QALY gained or VTE avoided. Prophylaxis was provided for 30 days. Outcomes and costs were half-cycle corrected and discounted at $3.5 \%$ (USA) and 5.0\% (Australia) per annum. In the USA,

Table 2 Cost and quality of life associated with VTE adverse events

\begin{tabular}{llll}
\hline Events & Cost in USA (USD) & Cost in Australia (AUD) & Annual quality of life disutility \\
\hline DVT & $18,104^{23}$ & $3,643^{\mathrm{a}}$ & $0.01(0.001)^{12}$ \\
PE & $18,104^{23}$ & $9,441^{\mathrm{a}}$ & $0.03(0.003)^{12}$ \\
DVT and PE & $37,290.67^{39}$ & $16,456^{\mathrm{a}}$ & $0.01(0.001)^{12}$ \\
Recurrent VTE & $27,955.87^{40}$ & $7,015^{\mathrm{a}}$ & As DVT \\
Non-major bleed & $367.22^{41}$ & $2325^{\mathrm{a}}$ & 0 \\
ICH & $36,217.45^{20}$ & $26,839^{\mathrm{a}}$ & $0.6(0.06)^{19}$ \\
Other major bleed & $17,567.39^{20}$ & $4,719^{\mathrm{a}}$ & $0.01(0.001)^{13}$ \\
Superficial wound infection & $7,644.03^{40}$ & $6,997^{\mathrm{a}}$ & $0.05(0.005)^{42}$ \\
Deep wound infection & $28,075.43^{40}$ & $37,648^{\mathrm{a}}$ & $0.04(0.004)^{42}$ \\
Heparin-induced thrombocytopenia & $18,177.43^{31}$ & $1,690^{28}$ & $0.02(0.002)^{43, c}$ \\
Severe PTS, per year & $6,040.93^{44}$ & $9,315^{\mathrm{b}}$ & $0.09(0.01)^{14}$ \\
Mild PTS, per year & $1,327.83^{44}$ & $4,104^{\mathrm{b}}$ & $0.09(0.01)^{14}$ \\
\hline
\end{tabular}

Notes: ${ }^{a h t t p s: / / w w w . h p p a . g o v . a u / s i t e s / g / f i l e s / n e t 636 / f / p u b l i c a t i o n s / f i n a l ~ n e p / 6 ~ d e t e r m i n a t i o n . p d f ; ~} 52$ bdata provided Australia AR-DRG, http://www.aihw.gov.au/hospitalsdata/ar-drg-data-cubes/; 53 creference indicates that HIT is associated with an extra 12.5 days in hospital. I day of full QoL is I/365.25 ( 0.0027378$)$ and assume $50 \%$ reduction in QoL (0.001369), multiplied by $12.5=0.017$.

Abbreviations: AR-DRG, Australian refined diagnosis-related group; DVT, deep vein thrombosis; HIT, heparin-induced thrombocytopenia; ICH, intracranial hemorrhage; PE, pulmonary embolism; PTS, postthrombotic syndrome; QoL, quality of life; RR, risk ratio; VTE, venous thromboembolism. 
the patient population had mean (SD) characteristics of age 63.17 (10.4) years, gender 41.2\% male, BMI 31.98 (7.03) $\mathrm{kg} / \mathrm{m}^{2}$, history of VTE $4.1 \%$, and 3 days in hospital. In Australia, these mean characteristics were age 67.5 (11.0) years, gender $40.5 \%$ male, BMI $31.0(7.03) \mathrm{kg} / \mathrm{m}^{2}$, history of VTE $7.7 \%$, and 5 days in hospital.

\section{Sensitivity analyses}

Probabilistic sensitivity analyses and scenario analyses were performed to explore the uncertainty around model outcomes. Probabilistic analysis used 500 runs of the model, in each run the value of each model input is varied at random and the model outputs extracted. A seeded random was used to allow for model verification and duplication. The random number drawn followed a uniform distribution from 0 to 1 and was used as a sample input variable against an underlying distribution by providing a random point on the cumulative probability curve. Most parameters were sampled against a normal distribution, but risk ratios used lognormal sampling. Results of probabilistic analyses are the cost-effectiveness plane, likelihood of a VTE prophylaxis method being considered cost-effective, and 95\% credible intervals (CrIs) around costs and QALYs. The willingness-to-pay thresholds were set to 50,000 AUD, and 100,000 USD, in line with the published recommendations as no guideline values exist. ${ }^{45,46}$

Scenario analyses considered outcomes in potential specialist patient populations and in hypothetical situations. Examples include patients at low or high risk of bleeding, those $<50$ years, and situations where VTE has no impact on QoL.

\section{Results}

For every 2,000 patients receiving LMWH after lower-limb arthroplasty in the USA, the model estimated that there would be 151 VTE events (including recurrence) and 6 major bleeding events in over 50 years of simulation (Table 3 ). Of these, 70 VTE events and 6 major bleeding events occurred in the first 6 months. Patients were expected to live a further 16.8 years on average, with 11.3 QALYs. The total health care cost per patient of using LMWH for VTE prophylaxis was estimated at 2,083 USD. Savings with IPC (426 USD) were larger than those with IPC+LMWH (133 USD), even though IPC+LMWH showed greater reduction in adverse events (15 VTEs) than did IPC (11 VTEs and 1 major bleed).

In the Australian setting, the number of VTE events (160) was similar but major bleeds (46) per 2,000 patients were much more common (Table 3). Notably, 152 of the VTE events occurred within the first 6 months. On average, life expectancy was 14.4 years, with 9.1 QALYs. The total health care cost per patient of using LMWH for VTE prophylaxis was estimated at 1,605 AUD. The use of IPC reduced the costs by 377 AUD and adverse events by 18 VTEs and 7 major bleeds. Use of IPC+LMWH in Australia reduced costs by 107 AUD and adverse events by 33 VTEs compared with LMWH alone.

Both IPC and IPC+LMWH resulted in more patient QALYs than did prophylaxis with LMWH alone (Table 3). Given the cost savings associated with both the forms of IPC prophylaxis, this made both IPC and IPC+LMWH dominant compared to LMWH alone (both for QALYs and VTE events). For IPC+LMWH relative to IPC alone, the combination increases the cost of care and the number of QALYs obtained. The incremental cost-effectiveness ratio (ICER) was 42,323 USD and 15,608 AUD per QALY gained, both about $50 \%$ below the willingness-to-pay threshold of the countries. The cost per VTE event avoided with IPC+LMWH versus IPC alone was 144,675 USD and 32,908 AUD in the

Table 3 Clinical and economic outcomes estimated by the models

\begin{tabular}{|c|c|c|c|c|c|c|}
\hline \multirow[t]{2}{*}{ Outcomes } & \multicolumn{2}{|l|}{ LMWH } & \multicolumn{2}{|l|}{ IPC } & \multicolumn{2}{|c|}{ IPC+LMWH } \\
\hline & USA & Australia & USA & Australia & USA & Australia \\
\hline Cost (USD / AUD) & 2,083 & 1,605 & 1,657 & 1,228 & 1,950 & $\mathrm{I}, 48 \mathrm{I}$ \\
\hline VTE (N per 2,000$)$ & $|5|$ & 160 & 140 & 142 & 136 & 127 \\
\hline Major bleeds ( $\mathrm{N}$ per 2,000$)$ & 6 & 46 & 5 & 39 & 6 & 46 \\
\hline Life years (mean) & 16.84 & 14.35 & 16.90 & 14.47 & 16.91 & $|4.5|$ \\
\hline QALYs (mean) & 11.28 & 9.07 & 11.32 & 9.14 & 11.33 & 9.15 \\
\hline \multicolumn{7}{|l|}{ ICER vs LMWH } \\
\hline Cost per QALY gained & - & - & Dominant & Dominant & Dominant & Dominant \\
\hline Cost per VTE avoided & - & - & Dominant & Dominant & Dominant & Dominant \\
\hline \multicolumn{7}{|l|}{ ICER vs IPC } \\
\hline Cost per QALY gained & Dominated & Dominated & - & - & 42,323 & 15,608 \\
\hline Cost per VTE avoided & Dominated & Dominated & - & - & 144,675 & 32,908 \\
\hline
\end{tabular}

Abbreviations: ICER, incremental cost-effectiveness ratio; IPC, intermittent pneumatic compression; LMWH, low-molecular-weight heparin; QALY, quality-adjusted life year; VTE, venous thromboembolism. 
USA and Australia, respectively. Both were considered above the expected willingness-to-pay threshold. ${ }^{45,46}$

If only significant differences between prophylaxis options and LMWH were modeled, then both IPC and IPC+LMWH remained dominant compared with LMWH alone. The ICER for IPC+LMWH versus IPC alone, though, decreased to 8,425 USD and 3,258 AUD per QALY gained in the USA and Australia, respectively (Table 4). Considering VTE, IPC alone resulted in more VTE events than LMWH if only significant differences were modeled. Health care payers were, though, estimated to save over 121.3 million USD and 9.5 million AUD per additional VTE event if IPC was used instead of LMWH. IPC+LMWH remained dominant to LMWH: fewer VTE events at lower cost. In all cases, the absolute differences per patient in QALYs and VTE events between prophylaxis options were small and warranted exploration in sensitivity analysis. The same is true when cost-effectiveness is considered as the cost per VTE event avoided.

\section{Sensitivity analyses}

Varying all model input parameters in line with their published uncertainty estimates determined that VTE prophylaxis using IPC was cost-effective in $74.25 \%$ and $97.60 \%$ of cases in the USA and Australia, respectively. For IPC+LMWH, the results were $92.02 \%$ and $97.80 \%$, respectively. The cost-effectiveness planes are presented in Figure 2. When prophylaxis with IPC+LMWH was compared with IPC alone, it was likely to be considered a cost-effective alternative in $37.13 \%$ and $15.57 \%$ of cases in the USA and Australia, respectively. The reason for this difference is likely due to the higher rates of bleeding reported in Australia, for which IPC will be a more attractive option given its low risk of bleeding (Table 1). From sensitivity analyses, IPC was found to increase patient QoL in $71.5 \%$ and $95.6 \%$ of simulations in the USA and Australia, respectively (Figures 3 and 4). Increased QoL was more consistent when IPC+LMWH was used and occurred in $91.2 \%$ and $93.2 \%$ of simulations, respectively. However, in every case, the $95 \% \mathrm{CrI}$ crossed zero, indicating a nonsignificant change. For costs, both IPC alone and IPC+LMWH showed a significant reduction in Australia. The median (95\% CrI) savings were 661 AUD (205-1,131) and 414 AUD (173-895) per patient for IPC and IPC+LMWH, respectively (Figures 3 and 4). In the USA, $90.6 \%$ and $96.4 \%$ of simulations resulted in reduced costs of care with IPC and IPC+LMWH, respectively, but neither reached significance.

Table 4 Results from the scenario analyses

\begin{tabular}{|c|c|c|c|c|c|c|}
\hline \multirow{2}{*}{$\begin{array}{l}\text { Scenario } \\
\text { comparison }\end{array}$} & \multicolumn{3}{|c|}{ Cost per QALY gained in the US } & \multicolumn{3}{|c|}{ Cost per QALY gained in Australia } \\
\hline & $\begin{array}{l}\text { IPC vs } \\
\text { LMWH }\end{array}$ & $\begin{array}{l}\text { IPC+LMWH } \\
\text { vs LMWH }\end{array}$ & $\begin{array}{l}\text { IPC+LMWH } \\
\text { vs IPC }\end{array}$ & $\begin{array}{l}\text { IPC vs } \\
\text { LMWH }\end{array}$ & $\begin{array}{l}\text { IPC+LMWH } \\
\text { vs LMWH }\end{array}$ & $\begin{array}{l}\text { IPC+LMWH } \\
\text { vs IPC }\end{array}$ \\
\hline \multicolumn{7}{|l|}{ Time horizon } \\
\hline 10 & Dominant & Dominant & 117,760 & Dominant & Dominant & 38,542 \\
\hline 20 & Dominant & Dominant & 52,157 & Dominant & Dominant & 17,556 \\
\hline 30 & Dominant & Dominant & 43,097 & Dominant & Dominant & 15,677 \\
\hline 40 & Dominant & Dominant & 42,339 & Dominant & Dominant & 15,610 \\
\hline \multicolumn{7}{|l|}{ Bleeding risk } \\
\hline Minor $10 \%$, major $2 \%$ & Dominant & Dominant & Dominated & Dominant & Dominant & $|7,04|$ \\
\hline Minor $2 \%$, major $0.4 \%$ & Dominant & Dominant & $55,5 \mid 4$ & Dominant & Dominant & 2,177 \\
\hline Minor I\%, major $0.01 \%$ & Dominant & Dominant & 23,455 & Dominant & Dominant & 914 \\
\hline \multicolumn{7}{|l|}{ Patient population } \\
\hline Aged 45 years & Dominant & Dominant & 24,165 & Dominant & Dominant & 7,520 \\
\hline Aged 75 years & Dominant & Dominant & 118,683 & Dominant & Dominant & 36,444 \\
\hline All TKA & Dominant & Dominant & 37,687 & Dominant & Dominant & 10,420 \\
\hline All THA & Dominant & Dominant & Dominated & Dominant & Dominant & Dominated \\
\hline \multicolumn{7}{|l|}{ Quality of life } \\
\hline No VTE decrement & Dominant & Dominant & 50,471 & Dominant & Dominant & 15,689 \\
\hline No fear of bleeding with LMWH & Dominant & Dominant & 27,402 & Dominant & Dominant & II,983 \\
\hline No major bleed decrement & Dominant & Dominant & 49,813 & Dominant & Dominant & $|5,50|$ \\
\hline Minor bleed at $0.00 \mathrm{I}$ & Dominant & Dominant & 50,535 & Dominant & Dominant & 15,674 \\
\hline Only sig. differences plus... & Dominant & Dominant & 8,425 & Dominant & Dominant & 3,258 \\
\hline ... Minor I\%, major $0.01 \%$ & I.I million SPQL & Dominant & 6,605 & $578,403 \mathrm{SPQL}$ & Dominant & Dominant \\
\hline ... DVT 10\% & Dominant & Dominant & Dominant & Dominant & Dominant & 114 \\
\hline
\end{tabular}

Notes: Dominant, more QALYs at lower cost; dominated, fewer QALYs at higher cost.

Abbreviations: DVT, deep vein thrombosis; IPC, intermittent pneumatic compression; LMWH, low-molecular-weight heparin; sig, significant; SPQL, saving per QALY lost; QALY, quality-adjusted life year; THA, total hip arthroplasty; TKA, total knee arthroplasty; VTE, venous thromboembolism. 


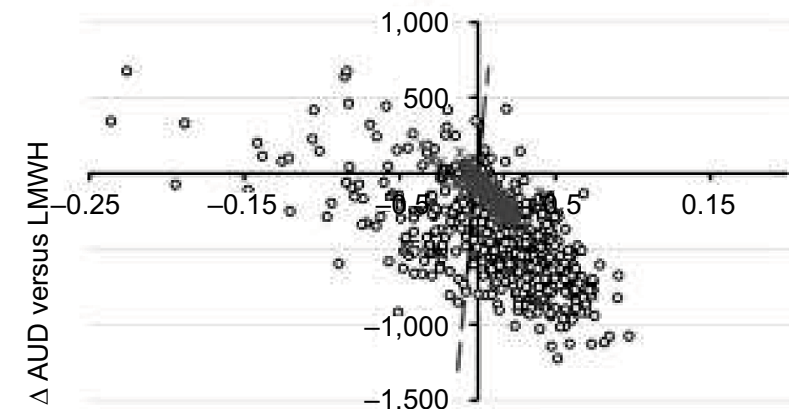

$\triangle$ QALYs versus LMWH

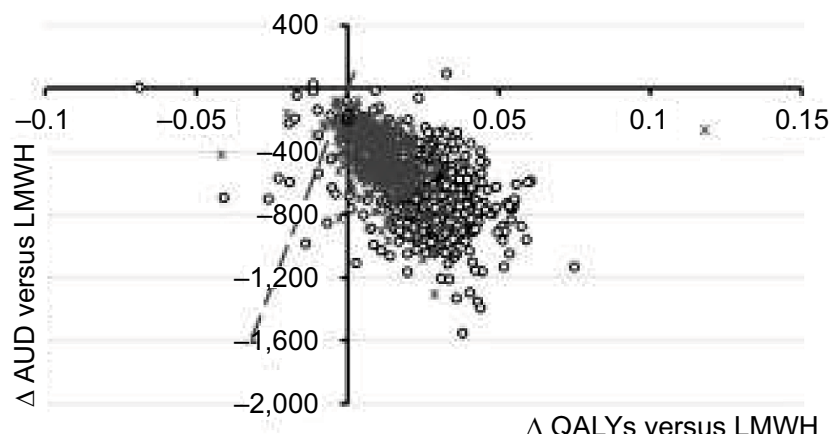

$\triangle \mathrm{QALYs}$ versus LMWH

- IPC $\quad$ IPC+LMWH - CE threshold

Figure 2 Cost-effectiveness plane for IPC and IPC+LMWH compared with LMWH alone.

Notes: The cost-effectiveness plane for IPC (open circles) and IPC+LMWH (gray stars) compared with LMWH alone in the USA (left) and Australia (right). The gray dashed line indicates the willingness-to-pay threshold, with points that are below and to the right of the line considered cost-effective.

Abbreviations: IPC, intermittent pneumatic compression; LMWH, low-molecular-weight heparin; QALY, quality-adjusted life year.

A

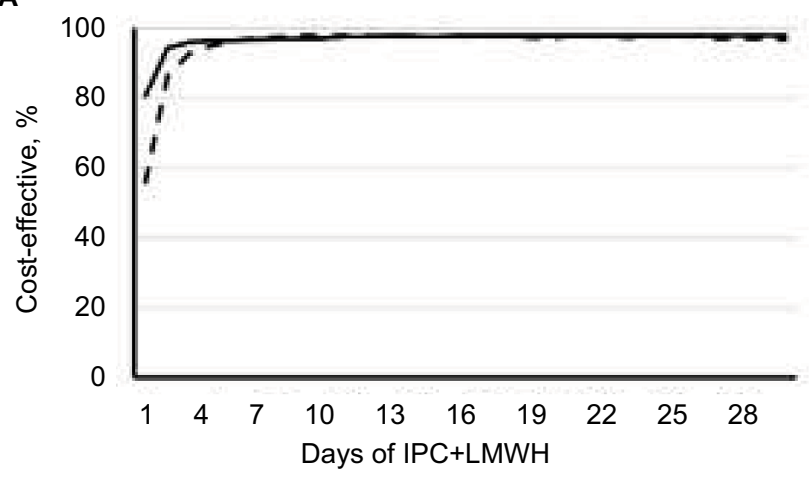

B

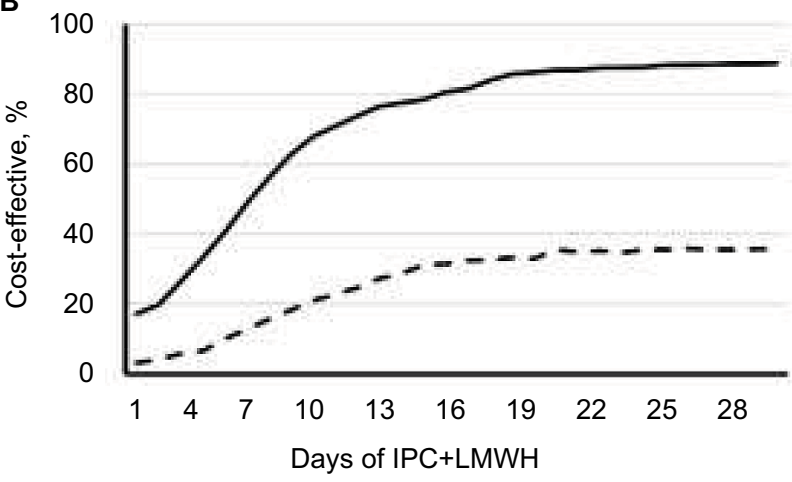

US $=$ - Aus

Figure 3 Likelihood of cost-effectiveness of IPC+LMWH by the number of days of combined use.

Notes: In the base case, IPC+LMWH is used while in hospital (3 days in USA and 5 days in Australia) followed by LMWH after discharge. As the number of days that IPC+LMWH is used, the likelihood of it being cost-effective increases. By 7 days, it is extremely likely to be considered cost-effective versus $\mathrm{LMWH}$ (A) but $<50 \%$ of simulations would be considered cost-effective versus IPC alone (B). All data from 500 sensitivity analyses were performed for each length of combined IPC+LMWH prophylaxis.

Abbreviations: Aus, Australia; IPC, intermittent pneumatic compression; LMWH, low-molecular-weight heparin.
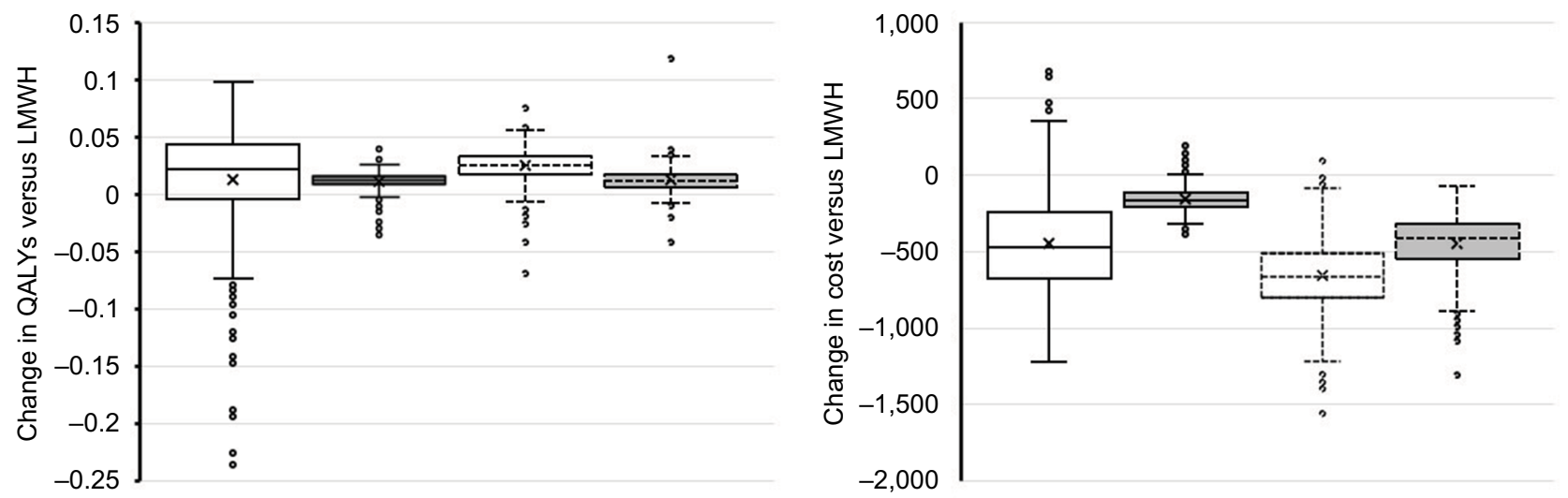

Figure 4 Uncertainty in model outcomes.

Notes: Box plots show the distribution of data from 500 sensitivity analyses, with IPC depicted in white and IPC+LMWH in gray. Data for the USA have a solid black outline, whereas Australian data have a dashed-black outline.

Abbreviations: IPC, intermittent pneumatic compression; LMWH, low-molecular-weight heparin; QALY, quality-adjusted life year. 
The impact of bleeding rates on outcomes and the relative effectiveness and cost-effectiveness of prophylaxis outcomes is clear from scenario analyses. If bleeding is relatively common, then IPC dominates (incurs lower costs and improves QALYs) the combination therapy of IPC+LMWH (Table 4). As bleeding events become less frequent, IPC+LMWH becomes a more cost-effective alternative. Similarly, younger patients and a longer time horizon are factors found to increase the cost-effectiveness of IPC+LMWH versus IPC alone. In general, both IPC and IPC+LMWH dominate LMWH alone. In exploratory analyses, in situations in which only significant differences were modeled and bleeding risk was low, did IPC alone not dominate LMWH alone. In this case, IPC alone was cost-saving but resulted in reduced patient QoL (Table 4). Given scenario analyses that were performed and that in general more VTE than bleeding events are prevented, it is likely that bleeding is a key driver of cost-effectiveness in VTE prophylaxis.

IPC+LMWH can vary in its use of the two components, in this analysis it is taken to be the combination of IPC+LMWH while the patient is in hospital ( 3 days in USA and 5 days in Australia) followed by LMWH alone up to day 30. If the number of days for which the combination is used before switching to LMWH alone is varied, the likelihood of IPC+LMWH being considered cost-effective changes. In general, the more number of days for which the combination is used, the higher the percentage of simulations considered cost-effective (Figure 2). Compared with LMWH alone, IPC+LMWH was found to be cost-effective in $97.0 \%$ and 97.4\% of simulations in the USA and Australia, if used for 7 days. Relative to IPC alone, this was $56.3 \%$ and $15.8 \%$ of simulations.

\section{Discussion}

Prophylaxis for VTE prevention after orthopedic surgery is predominantly performed using LMWH. ${ }^{9}$ However, the risk of bleeding events with LMWH means that it is often contraindicated in patients at high risk of hemorrhage.$^{47}$ Identifying these patients is challenging, and studies have shown that it cannot be predicted with accuracy in patients at risk for VTE. ${ }^{48,54}$ Minimizing risk of harms from increased risk of bleeding by use of mechanical means to prevent VTE makes sense; hence, finding a prophylaxis strategy that balances the risks of VTE events and bleeding is a priority.

Our analysis investigated whether IPC or IPC combined with LMWH (IPC+LMWH) may be more cost-effective strategies for VTE prophylaxis than LMWH alone. In line with published literature, our results showed that compared with LMWH, IPC alone is not inferior in preventing DVT and $\mathrm{PE}$, but it can significantly reduce minor and major bleeding events. ${ }^{22,29}$ On the other hand, IPC+LMWH has demonstrated significant reductions in DVT and PE, but there is little evidence of its impact on bleeding. ${ }^{22}$ In a 2017 neurosurgical study, postoperative IPC+LMWH use was found to reduce more than half the risk of DVT compared to LMWH and elastic stockings. ${ }^{49}$ Over a 50 -year time horizon, our analysis determined that both IPC alone and IPC+LMWH were likely to be cost-effective alternatives to VTE prophylaxis with LMWH. IPC alone was generally the least costly prophylaxis strategy, whereas IPC+LMWH generally resulted in the highest level of patient QoL. These results reflect mean overall outcomes of many patients; at the patient level, the most cost-effective strategy is likely to vary depending on individual's risk of VTE and bleeding.

Peer-reviewed data identified for model inputs indicated that major bleeding (defined as gastrointestinal or ICH) had a higher impact on patient $\mathrm{QoL}$ and was costlier to treat than both DVT and PE (Table 2). The same is true of long-term care costs. Here, though, major bleeding events more often result in mortality (both directly and within the next years) than does VTE, and patients who develop PTS can have high care costs for a long period of time. The drivers of cost, therefore, appear to be the relative incidence of major bleeding and VTE events. Scenario analyses showed that as bleeding risk decreased, IPC+LMWH became a more cost-effective alternative to IPC alone. Likewise, if only significant differences in prophylaxis options were modeled, IPC+LMWH dominated IPC alone if VTE rates were high. Model results, therefore, may indicate that in patients at high risk of bleeding, IPC may be the most effective form of VTE prophylaxis. If bleeding risk is low or outweighed by the risk of VTE, then IPC+LMWH may be most beneficial. For both the options with IPC, it is likely that care costs will be reduced.

The results from this analysis are in line with previous estimates. The Markov model by Tabatabaee et al considered VTE prophylaxis after total joint arthroplasty with either warfarin or aspirin. ${ }^{50}$ In this study, the cost of care in patients with a mean age of 65 years was 1,568-2,655 USD and patient had, on average, 10.3 QALYs. ${ }^{50}$ Zindel et al considered the cost-effectiveness of VTE prophylaxis following major orthopedic surgery in the Netherlands and found that prophylaxis with LMWH cost 1,754 GBP and resulted in 9.02 QALYs. ${ }^{51}$ In 2010, Kapoor et al systematically reviewed the literature available on cost-effectiveness of VTE prophylaxis following hip and knee surgery. ${ }^{52}$ The authors determined that ICER with LMWH was 312-109,000 USD per VTE event avoided.52 
Our VTE and major bleed incidences are in line with those identified by Kapoor et al for LMWH: DVT (1.1\%-20.8\%), PE (0\%-2.3\%), and major bleeds $(0 \%-6.6 \%) .{ }^{52}$ Varying the VTE and major bleeding rates in our model did change cost and QALY outcomes, but did not alter the conclusions drawn. All models are, however, only a representation of real-life practice and provide a likely estimate. Prospective clinical studies with cost collection are required to demonstrate the cost of care in real life and allow for comparison with model estimates. However, these are unavailable and are not feasible over the life time of enrolled patients.

Our model results indicate that the most cost-effective prophylaxis strategy is likely both patient and context dependent. Scenario analyses suggest that immediately after surgery, IPC alone is beneficial in patients at high risk of bleeding such as after major revision surgery, whereas IPC+LMWH may be more appropriate for patients at high VTE risk and low bleeding risk including those who have a history of VTE. Perhaps, a flexible combined use of IPC and LMWH, sequentially according to the bleeding risk after surgery will be the most appropriate approach to maximize the cost-effectiveness of VTE prophylaxis.

\section{Conclusion}

Both IPC alone and combining IPC with LMWH are more cost-effective than LMWH alone after lower-limb arthroplasty in both the US and Australian health care settings. From a clinician's and patient's perspective, the choice between IPC and IPC+LMWH likely depends on whether the risk of VTE outweighs the risk of major bleeding. IPC is the most suitable means of VTE prophylaxis in highbleeding-risk patients, whereas IPC+LMWH reduces VTE events and is most appropriate for patients at low bleeding risk and high risk of VTE. Individualizing the sequential use of IPC and LMWH to prevent VTE after arthroplasty should be seriously considered.

\section{Acknowledgment}

This work was funded by Medtronic.

\section{Disclosure}

RS is the owner and RTT an employee of Coreva Scientific $\mathrm{GmbH} \& \mathrm{Co} \mathrm{KG}$, which received consultancy fees for performing, analyzing, and communicating the work presented here. $\mathrm{AO}$ is a former employee of Medtronic. KMH and AJC are medical practitioners who have previously advised for Covidien, Medtronic, and other pharmaceutical and medical device companies. They did not receive remuneration for their work on this research. The authors report no other conflicts of interest in this work.

\section{References}

1. Jha AK, Larizgoitia I, Audera-Lopez C, Prasopa-Plaizier N, Waters H, Bates DW. The global burden of unsafe medical care: analytic modelling of observational studies. BMJ Qual Saf. 2013;22(10):809-815.

2. Deitelzweig SB, Johnson BH, Lin J, Schulman KL. Prevalence of clinical venous thromboembolism in the USA: current trends and future projections. Am J Hematol. 2011;86(2):217-220.

3. Grosse SD, Nelson RE, Nyarko KA, Richardson LC, Raskob GE. The economic burden of incident venous thromboembolism in the United States: a review of estimated attributable healthcare costs. Thromb Res. 2016;137:3-10.

4. Barco S, Woersching AL, Spyropoulos AC, Piovella F, Mahan CE. European Union-28: an annualised cost-of-illness model for venous thromboembolism. Thromb Haemost. 2016;115(4):800-808.

5. Australian Institute of Health and Welfare. OECD health-care quality indicators for Australia 2015; 2016. Available from https://www.aihw. gov.au/reports/international-comparisons/oecd-health-care-qualityindicators-2015/contents/table-of-contents. Accessed January 31, 2017

6. Access Economics. The burden of venous thromboembolism in Australia. Report for the Australia and New Zealand Working Party on the Management and Prevention of Venous Thromboembolism. 2008.

7. Charters MA, Frisch NB, Wessell NM, Dobson C, Les CM, Silverton CD. Rivaroxaban versus enoxaparin for venous thromboembolism prophylaxis after hip and knee arthroplasty. $J$ Arthroplasty. 2015;30(7):1277-1280.

8. Assareh H, Chen J, Ou L, Hollis SJ, Hillman K, Flabouris A. Rate of venous thromboembolism among surgical patients in Australian hospitals: a multicentre retrospective cohort study. BMJ Open. 2014;4(10):e005502.

9. Mirkazemi C, Bereznicki LR, Peterson GM. Thromboprophylaxis following hip and knee arthroplasty. Intern Med J. 2013;43(2):124-129.

10. Australian Orthopaedic Association National Joint Replacement Registry. 2016 NJRR Annual Report. Available from: https://aoanjrr.sahmri. com/de/annual-reports-2016. Accessed January 31, 2017.

11. Peel TN, Cheng AC, Liew D, et al. Direct hospital cost determinants following hip and knee arthroplasty. Arthritis Care Res (Hoboken). 2015;67(6):782-790.

12. Coleman CI, Limone BL, Bookhart BK, Mody SH, Nutescu EA. Cost-effectiveness analysis of extended duration anticoagulation with rivaroxaban to prevent recurrent venous thromboembolism. Thromb Res. 2014;133(5):743-749.

13. Hogg K, Kimpton M, Carrier M, Coyle D, Forgie M, Wells P. Estimating quality of life in acute venous thrombosis. JAMA Intern Med. 2013;173(12):1067-1072.

14. Enden T, Wik HS, Kvam AK, Haig Y, Kløw NE, Sandset PM. Healthrelated quality of life after catheter-directed thrombolysis for deep vein thrombosis: secondary outcomes of the randomised, non-blinded, parallel-group CaVenT study. BMJ Open. 2013;3(8):e002984.

15. Falck-Ytter Y, Francis CW, Johanson NA, et al. Prevention of VTE in orthopedic surgery patients. Antithrombotic therapy and prevention of thrombosis, 9th ed: American College of Chest Physicians evidence-based clinical practice guidelines. Chest. 2012;141(2 Suppl):e278S-e325S.

16. Khot A, Polmear A. Venous thromboembolism. In: Practical General Practice. 6th ed. London: Elsevier; 2011:639-640.

17. King DAL, Pow RE, Dickison DM, Vale PR. Apixaban versus enoxaparin in the prevention of venous thromboembolism following total knee arthroplasty: a single-centre, single-surgeon, retrospective analysis Intern Med J. 2016;46(9):1030-1037. 
18. Fiessinger J-N, Huisman MV, Davidson BL, et al. Ximelagatran vs lowmolecular-weight heparin and warfarin for the treatment of deep vein thrombosis: a randomized trial. J Am Med Assoc. 2005;293(6):681-689.

19. Lee HY, Hwang JS, Jeng JS, Wang J Der. Quality-adjusted life expectancy (QALE) and loss of QALE for patients with ischemic stroke and intracerebral hemorrhage: a 13-year follow-up. Stroke. 2010;41(4): 739-744.

20. Mercaldi CJ, Siu K, Sander SD, et al. Long-term costs of ischemic stroke and major bleeding events among Medicare patients with nonvalvular atrial fibrillation. Cardiol Res Pract. 2012;1(1):645469.

21. Kakkos SK, Szendro G, Griffin M, Daskalopoulou SS, Nicolaides AN. The efficacy of the new SCD response compression system in the prevention of venous stasis. J Vasc Surg. 2000;32(5):932-940.

22. Ho KM, Tan JA. Stratified meta-analysis of intermittent pneumatic compression of the lower limbs to prevent venous thromboembolism in hospitalized patients. Circulation. 2013;128(9):1003-1020.

23. Shahi A, Chen AF, Tan TL, Maltenfort MG, Kucukdurmaz F, Parvizi J. The incidence and economic burden of in-hospital venous thromboembolism in the United States. J Arthroplasty. 2017;32(4):1063-1066.

24. Pow RE, Vale PR. Thromboprophylaxis in patients undergoing total hip and knee arthroplasty: a review of current practices in an Australian teaching hospital. Intern Med J. 2015;45(3):293-299.

25. Spyropoulos AC, Lin J. Direct medical costs of venous thromboembolism and subsequent hospital readmission rates: an administrative claims analysis from 30 managed care organizations. J Manag Care Pharm. 2007;13(6):475-486.

26. Joseph JE, Low J, Courtenay B, Neil MJ, McGrath M, Ma D. A singlecentre prospective study of clinical and haemostatic risk factors for venous thromboembolism following lower limb arthroplasty. $\mathrm{Br} J$ Haematol. 2005;129(1):87-92.

27. Nelson DW, Simianu V V, Bastawrous AL, et al. Thromboembolic complications and prophylaxis patterns in colorectal surgery. JAMA Surg. 2015;150(8):712-720.

28. Duff J, Walker K, Omari A, Stratton C. Prevention of venous thromboembolism in hospitalized patients: analysis of reduced cost and improved clinical outcomes. J Vasc Nurs. 2013;31(1):9-14.

29. O'Connell S, Bashar K, Broderick BJ, et al. The use of intermittent pneumatic compression in orthopedic and neurosurgical postoperative patients. Ann Surg. 2016;263(5):888-889.

30. Linkins L, O'donnell M, Julian JA, Kearon C. Intracranial and fatal bleeding according to indication for long-term oral anticoagulant therapy. J Thromb Haemost. 2010;8(10):2201-2207.

31. Smythe MA, Koerber JM, Mattson JC. The incidence of recognized heparin-induced thrombocytopenia in a large, tertiary care teaching hospital. Chest. 2007;131(6):1644-1649.

32. Prandoni P, Lensing AW, Cogo A, et al. The long-term clinical course of acute deep venous thrombosis. Ann Intern Med. 1996;125(1):1-7.

33. Kahn SR, Galanaud J-P, Vedantham S, Ginsberg JS. Guidance for the prevention and treatment of the post-thrombotic syndrome. J Thromb Thrombolysis. 2016;41(1):144-153.

34. Briggs AH, Weinstein MC, Fenwick EAL, Karnon J, Sculpher MJ, Paltiel AD. Model parameter estimation and uncertainty: a report of the ISPOR-SMDM modeling good research practices task force-6. Value Health. 2012;15(6):835-842.

35. Caro JJ, Briggs AH, Siebert U, Kuntz KM. Modeling good research practices - overview: a report of the ISPOR-SMDM modeling good research practices task force-1. Value Health. 2012;15(6):796-803.

36. Siebert U, Alagoz O, Bayoumi AM, et al. State-transition modeling: a report of the ISPOR-SMDM modeling good research practices task force-3 background to the task force. Value Health. 2012;15(6):812-820.
37. Zhang J, Chen Z, Zheng J, Breusch SJ, Tian J. Risk factors for venous thromboembolism after total hip and total knee arthroplasty: a metaanalysis. Arch Orthop Trauma Surg. 2015;135(6):759-772.

38. Nichols CI, Vose JG. Clinical outcomes and costs within 90 days of primary or revision total joint arthroplasty. J Arthroplasty. 2016;31(7): 1400-1406.e3.

39. Fernandez MM, Hogue S, Preblick R, Kwong WJ. Review of the cost of venous thromboembolism. Clinicoecon Outcomes Res. 2015;7:451-462.

40. Schweizer ML, Cullen JJ, Perencevich EN, Vaughan Sarrazin MS. Costs associated with surgical site infections in Veterans Affairs Hospitals. JAMA Surg. 2014;52246(6):1-7.

41. Bullano MF, Willey V, Hauch O, Wygant G, Spyropoulos AC, Hoffman L. Longitudinal evaluation of health plan cost per venous thromboembolism or bleed event in patients with a prior venous thromboembolism event during hospitalization. J Manag Care Pharm. 2005;11(8):663-673.

42. Backes M, Schep NWL, Luitse JSK, Carel Goslings J, Schepers T. The effect of postoperative wound infections on functional outcome following intra-articular calcaneal fractures. Arch Orthop Trauma Surg. 2015;135(8):1045-1052.

43. Creekmore FM, Oderda GM, Pendleton RC, Brixner DI. Incidence and economic implications of heparin-induced thrombocytopenia in medical patients receiving prophylaxis for venous thromboembolism. Pharmacotherapy. 2006;26(10):1438-1445.

44. Caprini JA, Botteman MF, Stephens JM, et al. Economic burden of longterm complications of deep vein thrombosis after total hip replacement surgery in the United States. Value Health. 2003;6(1):59-74.

45. Neumann PJ, Cohen JT, Weinstein MC. Updating cost-effectiveness the curious resilience of the \$50,000-per-QALY threshold. $N$ Engl J Med. 2014;371(9):796-797.

46. Paris V, Belloni A. Value in pharmaceutical pricing. OECD Health Working Papers, No. 63; 2013:1-74.

47. Amber R. ACP recommendations for VTE prophylaxis in hospitalized patients. Am Fam Physician. 2012;85(12):1204.

48. Riva N, Bellesini M, Di Minno MND, et al. Poor predictive value of contemporary bleeding risk scores during long-term treatment of venous thromboembolism. Thromb Haemost. 2014;112(3):511-521.

49. Chibbaro S, Cebula H, Todeschi J, et al. Evolution of Prophylaxis Protocols for Venous Thromboembolism in Neurosurgery: Results from a Prospective Comparative Study on Low-Molecular-Weight Heparin, Elastic Stockings, and Intermittent Pneumatic Compression Devices. World Neurosurg. 2017;109:e510-e516.

50. Mostafavi Tabatabaee R, Rasouli MR, Maltenfort MG, Parvizi J. Cost-effective prophylaxis against venous thromboembolism after total joint arthroplasty: warfarin versus aspirin. J Arthroplasty. 2015;30(2):159-164.

51. Zindel S, Stock S, Müller D, et al. Economic evaluation of dabigatran etexilate for the primary prevention of venous tromboembolic events following major orthopedic surgery in the Netherlands. Pharmacoeconomics. 2015;13(2):376-383.

52. Kapoor A, Chuang W, Radhakrishnan N, et al. Cost effectiveness of venous thromboembolism pharmacological prophylaxis in total hip and knee replacement: a systematic review. Pharmacoeconomics. 2010;28(7):521-538

53. National Efficient Price Determination 2016-17. Independent Hospital Pricing Authority; 2016. Available from https://www.ihpa.gov.au/sites/g/ files/net636/f/publications/final_nep16_determination.pdf. Accessed January 31,2017

54. Scherz N, Mean M, Limacher A, et al. Prospective, multicenter validation of prediction scores for major bleeding in elderly patients with venous thromboembolism. J Thromb Haemost. 2013;11(3):435-443. 
ClinicoEconomics and Outcomes Research is an international, peerreviewed open-access journal focusing on health technology assessment, pharmacoeconomics and outcomes research in the areas of diagnosis, medical devices, and clinical, surgical and pharmacological intervention. The economic impact of health policy and health systems organization also constitute important areas of coverage. The manuscript management system is completely online and includes a very quick and fair peer-review system, which is all easy to use. Visit http://www.dovepress.com/testimonials.php to read real quotes from published authors.

Submit your manuscript here: https://www.dovepress.com/clinicoeconomics-and-outcomes-research-journal 